\title{
Violência verbal contra indivíduos com transtorno mental no Brasil: prevalência e fatores associados
}

\author{
Verbal violence against mentally ill individuals in \\ Brazil: prevalence and associated factors \\ Helian Nunes de Oliveira', Carla Jorge Machado², Mark Drew Crosland Guimarães²
}

\section{INTRODUÇÃO}

Considera-se violência não somente o abuso físico ou sexual, mas também a violência psicológica, que envolve a agressão emocional e a verbal'. A violência verbal é o tipo mais frequente de violência psicológica e já foi avaliada em diversas populações, tais como as populações de crianças', gestantes², usuárias em serviços de saúde ${ }^{3}$, trabalhadores e estudantes'.

Não há estudos nacionais que avaliem a relação da violência verbal com transtornos mentais nos diversos tipos de serviços públicos de saúde mental. Este trabalho avalia a prevalência e os fatores associados aos autorrelatos de violência verbal contra os indivíduos com transtorno mental, estratificando por sexo, em serviços de saúde mental no Brasil.

\section{MÉTODOS}

Os dados utilizados provieram de estudo transversal multicêntrico nacional (Projeto PESSOAS - Pesquisa em Soroprevalência de Aids na Saúde Mental), realizado entre usuários de serviços de saúde mental no Brasil4,5. Resumidamente, em 2006, 2.475 usuários de serviços de saúde mental cadastrados no Ministério da Saúde foram selecionados aleatoriamente de 26 serviços públicos de saúde (11 hospitais psiquiátricos e 15 centros de atenção psicossocial - CAPS), por amostragem estratificada proporcional à distribuição de casos notificados de Aids por regiões geográficas do Brasil e locais de tratamento (hospitais e CAPS, excluindo unidades psiquiátricas em hospital geral, serviços de atenção ao álcool e outras drogas, CAPS-AD e residências terapêuticas). As cidades que participaram do estudo foram: Manaus (AM); Paraíso (TO); Natal (RN); Morada Nova (CE); Santiago e Capão do Leão (RS); Maringá (PR); Anápolis e Goiânia (GO); São José do Rio Pardo, Assis, Guarujá, Sorocaba, Franco da Rocha e Lins (SP); Betim, Formiga, Montes Claros, Neves, Belo Horizonte e Divinópolis (MG); Cantagalo e Carmo (RJ). Foram incluídos indivíduos com 18 anos de idade ou mais, em tratamento nos serviços públicos de saúde mental, capazes de compreender e assinar o Termo de Consentimento Livre e Esclarecido, excluindo-se, assim, pacientes agudamente incapazes ou com deficiência mental mais grave. A pesquisa foi aprovada pelos serviços participantes, pelo Comitê de Ética e Pesquisa da Universidade Federal de Minas Gerais (UFMG/ETIC:125/05) e pelo Conselho Nacional de Ética em Pesquisa (Conep) 592/2006.

1 Instituto Raul Soares, Fundação Hospitalar do Estado de Minas Gerais (FHEMIG), Escola de Saúde Pública do Estado de Minas Gerais (ESP/MG). 2 Universidade Federal de Minas Gerais (UFMG), Faculdade de Medicina, Departamento de Medicina Preventiva e Social. 
A violência verbal foi um autorrelato da experiência pessoal de ter sofrido agressão verbal alguma vez na vida, definida como ter sofrido alguma vez algum tipo de agressão verbal, ou seja, ameaça, humilhação, xingamento, ofensa ou constrangimento. Essa definição tem maior sensibilidade e apresentou confiabilidade razoável e validade de construto ${ }^{4,5}$. Para aqueles que relataram ter sofrido violência verbal, foram avaliados: quem perpetrou a violência verbal (parceiro íntimo, pais, parentes, conhecidos, estranhos, profissionais de saúde e outros) e onde ocorreu (ambiente doméstico, instituição, ruas, ou qualquer outro lugar). Os entrevistadores eram profissionais de saúde mental, com conhecimento e experiência sobre transtornos mentais e serviços públicos de atenção em saúde mental, treinados previamente. Todos os procedimentos, protocolos e questionários foram testados em um estudo piloto e avaliados quanto à confiabilidade ${ }^{4,5}$.

Características sociodemográficas, clínicas e psiquiátricas e comportamentos e situações de risco para HIV/DST foram obtidos por entrevista estruturada aplicada pelo profissional de saúde mental e por coleta em prontuários médicos. Para essa análise, foram selecionadas variáveis sociodemográficas: centro de recrutamento (hospital psiquiátrico; CAPS), idade em anos (18 a 40; mais de 40), cor da pele (branco; não branco), escolaridade em anos de estudo (< $5 ; 5$ ou mais), renda familiar no último mês em reais $(<R \$$ 350,00; maior ou igual a $\mathrm{R} \$ 350,00$ ), estado civil atual, morar sozinho atualmente, histórico de morar na rua, lugar atual de residência (casa/apartamento ou outros - hospital, casa de apoio, albergue, pensão, morador de rua). Quanto às características clínicas ou médicas, foram: história de internação em hospital psiquiátrico, idade da primeira internação psiquiátrica (< 18 ou 18 ou mais), história de diagnóstico de doença sexualmente transmissível, diagnóstico psiquiátrico principal. Finalmente, quanto aos comportamentos e situações de risco para infecção pelo HIV/Aids, foram avaliados: consumo de álcool na vida, uso de cocaína ou maconha na vida, idade da primeira relação sexual (< 16 ou igual a 16 ou mais), relações sexuais sob o uso de álcool ou drogas, uso irregular de preservativos em qualquer relação sexual, história de receber ou oferecer dinheiro por sexo, histórico de encarceramento.

O uso de preservativo foi definido como irregular quando o participante relatou que não ocorreu em todas as relações sexuais na vida. Para o diagnóstico psiquiátrico, os dados foram obtidos dos prontuários, classificados de acordo com a Classificação Internacional de Doenças (CID-10) e categorizados em: 1. psicoses (esquizofrenia e outras psicoses não bipolares); 2. transtornos bipolares, 3. transtornos depressivos; 4. transtornos de ansiedade; 5 . transtornos de uso de substâncias psicoativas; 6 . outros. Os diagnósticos 4 a 6 foram agrupados e considerados como referência, pois os serviços públicos de saúde estudados têm o objetivo de assistir a clientela mais grave.

\section{Análise}

A análise descritiva incluiu a frequência da violência verbal relatada, a descrição da fonte (perpetrador) e do lugar de sua ocorrência. O teste de qui-quadrado de Pearson foi utilizado para avaliar as diferenças de proporção. A magnitude das associações entre as variáveis explanatórias e o autorrelato de violência verbal foi estimada pelo odds ratio (OR), com um intervalo de $95 \%$ de confiança, estratificado por sexo. O potencial efeito independente das variáveis preditoras de violência verbal foi avaliado por meio de modelo de regressão logística, estratificado por sexo ${ }^{6}$. Foi considerado nível de significância de 0,05. A obtenção do modelo final utilizou a regressão logística multivariada com estratégia de deleção sequencial. As variáveis que na análise univariada apresentaram $\mathrm{p}<0,20$ foram selecionadas para iniciar o modelo, e somente as variáveis com $\mathrm{p}<0,05$ permaneceram no modelo final, cujo ajuste foi avaliado por teste de Hosmer-Lemeshow? ${ }^{7}$ O banco de dados foi gerado no software PARADOX ${ }^{\circledR} 9$ e a análise foi realizada com auxílio do pacote estatístico do software SAS ${ }^{\circledR}$ 9.0.

\section{RESULTADOS}

Foram recrutados 3.225 pacientes e, desses, $2.763(84,9 \%)$ foram considerados elegíveis e 492 (15,1\%) não foram considerados capazes de participar do estudo após avaliação preliminar padronizada e avaliação clínica. A maioria dos que não foram considerados capazes apresentou algum grau de desorientação no tempo ou espaço, desorganização do pensamento ou ideias ou algum nível de retardo mental. Dentre esses elegíveis, 2.475 (89,6\%) foram entrevistados e $288(10,4 \%)$ não participaram, principalmente por causa de recusa, de não terem sido localizados ou de não comparecerem à entrevista. Os indivíduos hospitalizados tiveram maior proporção de participação quando comparados àqueles em tratamento no CAPS $(p<0,001)$. Não foram observadas diferenças estatisticamente significativas quanto a sexo, idade, escolaridade ou diagnóstico psiquiátrico.

A análise descritiva revelou que, dentre os 2.475 entrevistados, $1.277(51,6 \%)$ eram mulheres. As mulheres, em comparação com os homens, apresentaram maiores proporções de procedência de famílias com baixa renda $(27,1 \%$ versus 17,8\%), diagnóstico de transtorno bipolar (12,4\% versus 5,3\%) ou depressão (19,4\% versus 5,8\%) e uso irregular de preservativos $(83,5 \%$ versus $76,8 \%)$. Os homens, contudo, apresentaram maior proporção das seguintes características sociodemográficas em comparação às mulheres: 18 a 40 anos de idade ( $51,9 \%$ versus $44,9 \%)$, não brancos ( $51,2 \%$ versus $46,0 \%)$, escolaridade menor que cinco anos $(52,1 \%$ versus $49,1 \%)$, solteiros (62,9\% versus $35,3 \%)$, histórico de ter morado na rua ( $21,5 \%$ versus $15,0 \%$ ) e viver sozinho atualmente (15,7\% versus $10,3 \%)$. Os homens também apresentaram maiores proporções de variá- 
veis clínicas psiquiátricas de severidade, comparativamente às mulheres: houve maior proporção de hospitalização durante o recrutamento da pesquisa (44,8\% versus $28,3 \%$ ), hospitalização psiquiátrica prévia ( $66,3 \%$ versus $50,8 \%)$, idade da primeira hospitalização psiquiátrica menor que 18 anos (11,3\% versus $7,9 \%)$, diagnóstico de psicose (55,5\% versus 40,4\%). Quanto aos comportamentos/situações de risco, esses também estiveram presentes em maior proporção entre os homens: consumo de álcool na vida (77,5\% versus 52,7\%), idade da primeira relação sexual inferior a 16 anos (52,8\% versus $43,9 \%)$, uso de maconha e cocaína na vida (34,4\% versus $11,2 \%)$, relações sexuais sob o uso de álcool ou drogas (37,8\% versus $17,2 \%)$, troca de dinheiro por relações sexuais (40,6\% versus 13,3\%) e histórico de encarceramento ( $41,2 \%$ versus $10,6 \%)$.

A prevalência global de violência verbal contra essa população foi de $68,4 \%(n=1.693)($ IC $95 \%=66,6 ; 70,2): 72,5 \%$ $(n=926)(I C 95 \%=70,0 ; 74,9)$ entre mulheres e 64,0\% ( $n=$ 767) $($ IC $95 \%=61,3 ; 66,7)$ entre homens.

As principais fontes de violência verbal contra mulheres e homens foram, respectivamente, pais (21,1\% e 16,9\%), parentes (45,5\% e $36,8 \%$ ), parceiros (38,6\% e 14,3\%), funcionários, profissionais de saúde e outros pacientes em instituições de saúde (10,0\% e 13,2\%), conhecidos (20,4\% e 33,6\%), desconhecidos (5,6\% e 13,2\%) e outros (17,4\% e $24,0 \%)$; a violência verbal ocorreu em casa $(73,4 \%$ e $48,2 \%)$, na rua (32,0\% e 29,3\%), no trabalho (7,2\% e 14,3\%) e em instituições de saúde (10,0\% e 13,2\%).

A análise multivariada indicou que, para as mulheres, estiveram associados independente e estatisticamente ( $p$ > $0,05)$ com violência verbal: histórico de ter morado na rua, morar atualmente em casa ou apartamento, histórico de DST, consumo de álcool na vida e uso irregular de preservativos na vida. Para os homens, estiveram positivamente associados: cinco ou mais anos de escolaridade, não ser sol- teiro atualmente, morar sozinho atualmente, histórico de ter morado na rua, morar atualmente em casa ou apartamento, histórico de DST, uso de álcool na vida, uso de drogas ilícitas na vida, iniciação precoce de relações sexuais, ter tido relações sexuais sob efeito de álcool/drogas, trocar dinheiro por sexo na vida e histórico de encarceramento (Tabela 1).

\section{DISCUSSÃO}

Este estudo mostrou que a violência verbal ocorreu em maior proporção entre as mulheres (72,5\%) comparativamente aos homens $(64,0 \%)$ e esteve associada para ambos os sexos a duas variáveis sociodemográficas: histórico de ter morado na rua e morar em casa ou apartamento atualmente, o que corrobora outros achados ${ }^{8,9}$. Com relação aos indivíduos com histórico de morar na rua, há evidência de que eles, especialmente aqueles com transtorno mental, sofrem maior exposição à violência ${ }^{10}$. Em estudo longitudinal com indivíduos com transtorno mental crônico, foi encontrado, ainda, para ambos os sexos, maior risco de sofrer violência quanto tinham contato menos frequente com a família, revelando a ausência do suporte social e sua associação com a violência ${ }^{10}$. Quanto ao ambiente doméstico, ressalta-se em maior proporção a violência contra as mulheres. Pessoas que convivem no ambiente doméstico (parceiro, pais e parentes), de fato, foram os principais perpetradores nos relatos de violência verbal contra as mulheres. Contudo, as mulheres também se encontram mais fragilizadas em outras esferas, pois a violência verbal contra as mulheres na rua foi relatada em maior proporção do que pelos homens. O achado generalizado de maior prevalência de violência contra as mulheres com transtorno mental é corroborado por outras análises ${ }^{11}$.

Tabela 1. Modelo multivariado estratificado por sexo para violência verbal contra indivíduos com transtorno mental (Projeto PESSOAS, Brasil, 2007)

\begin{tabular}{|c|c|c|c|c|}
\hline & \multicolumn{2}{|c|}{ Mulheres } & \multicolumn{2}{|c|}{ Homens } \\
\hline & ORaja $^{a}$ & $(\text { IC 95\%) })^{\mathrm{a}}$ & ORaja $^{\mathrm{a}}$ & $(\text { IC 95\% })^{\mathrm{a}}$ \\
\hline Escolaridade (5 ou mais anos de estudo) & - & & 1,55 & $(1,19-2,01)^{* *}$ \\
\hline Estado civil atual (não solteiro) & - & & 1,44 & $(1,09-1,88)^{* *}$ \\
\hline Mora sozinho(a) atualmente & - & & 1,53 & $(1,05-2,25)^{*}$ \\
\hline Ter morado na rua alguma vez na vida & 1,84 & $(1,18-2,86)^{* *}$ & 2,47 & $(1,69-3,60)^{* *}$ \\
\hline Onde mora atualmente (casa ou apartamento) & 2,38 & $(1,48-3,83)^{* *}$ & 1,63 & $(1,13-2,35)^{* *}$ \\
\hline História de diagnóstico de DST na vida & 2,07 & $(1,40-3,06)^{* *}$ & 1,49 & $(1,08-2,06)^{*}$ \\
\hline Consumo de álcool na vida & 1,82 & $(1,39-2,38)^{* *}$ & 1,42 & $(1,04-1,94)^{*}$ \\
\hline Uso irregular de preservativos na vida & 1,59 & $(1,14-2,21)^{* *}$ & - & \\
\hline Recebido ou oferecido dinheiro por sexo & - & & 1,33 & $(1,01-1,76)^{*}$ \\
\hline Histórico de encarceramento & - & & 1,34 & $(1,01-1,78)^{*}$ \\
\hline
\end{tabular}

a $=$ Odds Ratio ajustados e intervalos de $95 \%$ de confiança; ${ }^{*} p<0,05$. ${ }^{* *} p<0,01$.

Teste de Hosmer-Lemeshow (mulheres): $p=0,722 ;$ graus de liberdade $=5$.

Teste de Hosmer-Lemeshow (homens): $p=0,341$; graus de liberdade $=8$. 
A violência verbal foi associada com uso irregular de preservativos na vida apenas entre as mulheres, indicando vulnerabilidade associada ao gênero, ou como se organizam as relações sociais e o papel da mulher no contexto das relações, influenciando comportamentos de risco ${ }^{12}$.

A violência verbal contra os homens foi associada à escolaridade superior a cinco anos, não estar solteiro, morar sozinho atualmente, troca de dinheiro por sexo, histórico de encarceramento"1 e funcionamento social ruim ${ }^{11}$. Especificamente quanto à escolaridade, foi observado que a percepção da vulnerabilidade à violência dos indivíduos com transtorno mental apresentou-se baixa, porém os indivíduos com transtorno mental e maior nível de escolaridade apresentaram mais sentimentos de vulnerabilidade quanto à violência' ${ }^{13}$. Porém, nesses estudos não foi estudada especificamente a violência verbal ou psicológica e diferenças quanto ao sexo.

Este estudo apresenta limitações. Os dados são de 2006, havendo uma distância de sete anos entre a data da pesquisa e o ano corrente. Nesse período, ocorreram mudanças no contexto dos serviços públicos de saúde mental ou psiquiátricos. Não foram incluídos pacientes mais graves instáveis, com deficiência mental grave ou em tratamento em serviços específicos de atenção em dependência química. Isso pode ter subestimado a prevalência de violência verbal.

\section{CONCLUSÃO}

Foram encontrados índices de violência verbal bastante altos entre os pacientes com transtorno mental entre os homens $(64,0 \%)$, mas especialmente entre as mulheres (72,5\%), para as quais houve ainda alta proporção de violência no ambiente doméstico. Este trabalho também explicitou os fatores independentemente associados ao desfecho. O conhecimento dos fatores associados, segundo o sexo, reforça, para os profissionais que atuam em serviços de saúde mental, a necessidade de intervenções precoces na prevenção da violência verbal e de outras violências dela decorrentes, focalizadas nas necessidades de cada indivíduo e de suas famílias.

\section{CONTRIBUIÇÕES INDIVIDUAIS}

Todos os autores contribuíram significativamente na concepção e desenho dos estudos, na análise e interpretação dos dados, na elaboração do artigo e na revisão crítica do seu conteúdo intelectual. Todos os autores aprovaram a versão final a ser publicada.

\section{CONFLITOS DE INTERESSE}

Os autores declaram não haver conflitos de interesse de qualquer natureza que possam ter influenciado na interpretação dos resultados aqui apresentados ou comprometido a integridade da pesquisa.

\section{AGRADECIMENTOS/FINANCIAMENTO}

Este trabalho foi conduzido pela Universidade Federal de Minas Gerais, com o apoio técnico e financeiro do Ministério da Saúde/ Secretaria de Vigilância em Saúde/Departamento de DST, Aids e Hepatites Virais por meio do Projeto de Cooperação Técnica 914/BRA/1101 entre o governo brasileiro e a Organização das Nações Unidas para a Educação, a Ciência e a Cultura - Unesco.

\section{REFERÊNCIAS}

1. Krug EG, Dahlberg LL, Mercy JA, Zwi AB, Lozano R. World report on violence and health. Geneva: World Health Organization; 2002

2. Ludermir AB, Lewis G, Valongueiro SA, de Araújo TV, Araya R. Violence against women by their intimate partner during pregnancy and postnatal depression: a prospective cohort study. Lancet. 2010;11;376(9744):903-10.

3. Schraiber LB, D'Oliveira AF, Couto MT, Hanada H, Kiss LB, Durand JG, et al. Violence against women attending public health services in the metropolitan area of São Paulo, Brazil. Rev Saude Publica. 2007;41(3):359-67.

4. Guimarães MDC, Campos LN, Melo AP, Carmo RA, Machado CJ, Acurcio FA. PESSOAS Project Network Group. Prevalence of HIV, syphilis, hepatitis $B$ and $C$ among adults with mental illness: a multicenter study in Brazil. Rev Bras Psiquiatr. 2009;31(1):43-7.

5. Guimarães MDC, Oliveira HN, Campos LN, Santos CA, Gomes CER, Oliveira SB, et al. Reliability and validity of a questionnaire on vulnerability to sexuality transmitted infections among adults with chronic mental illness - PESSOAS Project. Rev Bras Psiquiatr. 2008:30(1):55-9.

6. Kahn HA, Sempos CT. Statistical methods in epidemiology. $2^{\text {nd }}$ ed. New York: Oxford University Press; 1997.

7. Hosmer DW, Lemeshow S. Applied logistic regression. New York: John Wiley \& Sons; 1989.

8. Walsh E, Moran P, Scott C, McKenzie K, Burns T, Creed F, et al.; UK700 Group. Prevalence of violent victimisation in severe mental illness. Br J Psychiatry. 2003;183:233-8.

9. Howard LM, Trevillion K, Khalifeh H, Woodall A, Agnew-Davies R, Feder G. Domestic violence and severe psychiatric disorders: prevalence and interventions. Psychol Med. 2010;40(6):881-93.

10. Dean K, Moran P, Fahy T, Tyrer P, Leese M, Creed F, et al. Predictors of violent victimization amongst those with psychosis. Acta Psychiatr Scand. 2007;116(5):345-53.

11. Chapple B, Chant D, Nolan P, Cardy S, Whiteford H, McGrath J. Correlates of victimisation amongst people with psychosis. Soc Psychiatry Psychiatr Epidemiol. 2004;39(10):836-40.

12. WHO. Addressing violence against women and HIV/AIDS: What works? Geneva: World Health Organization; 2010 .

13. Hiday VA, Swartz MS, Swanson JW, Borum R, Wagner HR. Criminal victimization of persons with severe mental illness. Psychiatr Serv. 1999;50(1):62-8. 